Revue européenne des sciences sociales

European Journal of Social Sciences

XXXVIII-117| 2000

Métaphores et analogies. Schèmes argumentatifs des sciences sociales

\title{
La métaphore du labyrinthe chez Kafka
}

Hervé Le Bras

\section{OpenEdition}

1 Journals

Édition électronique

URL : http://journals.openedition.org/ress/719

DOI : $10.4000 /$ ress. 719

ISSN : 1663-4446

Éditeur

Librairie Droz

Édition imprimée

Date de publication : 1 février 2000

Pagination : 159-171

ISBN : 2-600-00409-2

ISSN : 0048-8046

Référence électronique

Hervé Le Bras, «La métaphore du labyrinthe chez Kafka », Revue européenne des sciences sociales [En ligne], XXXVIII-117 | 2000, mis en ligne le 17 décembre 2009, consulté le 01 mai 2019. URL : http:// journals.openedition.org/ress/719; DOI : 10.4000/ress.719 


\section{Hervé LE BRAS}

\section{LA MÉTAPHORE DU LABYRINTHE CHEZ KAFKA}

Joseph K. est surpris quand au lieu du petit déjeuner apporté par la bonne Anna, apparaît dans l'embrasure de la porte un individu sanglé dans un curieux uniforme, venu lui signifier son arrestation. Dès les premières lignes du Procès, Joseph K. est confronté à l'énigme qu'il cherchera en vain à résoudre durant tout le roman: des fonctionnaires subalternes appliquent avec tous les gestes requis une procédure engagée contre lui, mais il ne parvient jamais à connaître le motif de son accusation. Dans le second chapitre du Procès, Joseph K., laissé en liberté provisoire, efface à la banque où il travaille les traces de son arrestation et rentre faire de même à la pension où il habite. Il attend longuement le retour d'une certaine $\mathbf{M}^{\text {lle }}$ Bürstner, dactylographe, qui occupe la chambre où son inculpation lui a été signifiée. Lorsqu'elle rentre du théâtre sur le coup de onze heures du soir, il demande à lui parler dans sa chambre pour lui expliquer les événements du matin, et peu à peu est attiré par elle. En la quittant, il la saisit, l'embrasse sur la bouche, sur tout le visage et goulûment sur la gorge, «à l'endroit du gosier». Dans le troisième chapitre, $M^{\text {lle }}$ Bürstner refuse les nouvelles explications que Joseph $K$. tente de lui donner, et ayant mis dans la confidence d'autres pensionnaires, se fait protéger par eux. Malgré cela, Joseph K. ne résiste pas au désir de revoir la chambre de $\mathrm{M}^{\mathrm{ll}}$ Bürstner. «Il ouvrit finalement la porte avec prudence, non sans éprouver le sentiment de commettre une faute, et qui pis est une faute inutile.» ${ }^{1}$ En sortant, il a l'impression que $\mathbf{M}^{\mathrm{lle}}$ Montag et le capitaine Lanz, deux amis de $\mathbf{M}^{\mathrm{lle}}$ Bürstner, l'ont vu.

On sait que Kafka n'avait pas indiqué dans ses cahiers l'ordre des chapitres de ses romans. Max Brod, son ami et exécuteur testamentaire le reconstitua pour le Procès. Il y eut une controverse ${ }^{2}$, mais aujourd'hui, tout le monde s'accorde sur la solution adoptée par Brod. Ceci montre cependant que Kafka gardait une certaine latitude pour l'ordre des chapitres. On peut contraster son hésitation à la certitude que, par exemple, Thomas Mann avait de la succession des chapitres du Docteur Faustus, avant même d'avoir écrit la première ligne ${ }^{3}$. Supposons alors que le premier chapitre du Procès, qui relate l'arrestation, soit déplacé en position de

Les citations des romans et histoires de Kafka sont extraites de l'édition intégrale de la Pléiade. Ici, T. I, p. 294.

2 Lancée par H. Uyttersprot (Eine neue Ordnung der Werke Kafkas? Zur Struktur von dem «Prozess» und «Amerika», Anvers, Nijhoff, 1957).

3 Il l'a montré dans le Journal du Docteur Faustus, dans lequel il a relaté la rédaction de son roman et les lectures publiques qu'il fit des chapitres successifs. 
troisième chapitre. Le second chapitre devient ainsi le premier et le troisième, le second. Une telle permutation fait perdre au roman son mystère et le rend quasiment transparent. En effet, la scène de la séduction de $\mathrm{M}^{\mathrm{lle}}$ Bürstner par laquelle commencerait le roman dans ces conditions, s'interprèterait comme une scène de viol. A un moment d'ailleurs, pour lui expliquer la scène de l'arrestation, Joseph K. mime devant $\mathbf{M}^{\text {lle }}$ Bürstner le greffier l'interpellant et crie «Joseph K.» d'une voix sourde qui alerte le capitaine Lanz, lequel peut penser que c'est $\mathbf{M}^{\text {lle }}$ Bürstner qui crie en se défendant contre les assauts de son violeur. Le baiser sur le gosier prend un caractère menaçant: si tu parles, je te tranche le cou. Dans le second chapitre de cette nouvelle construction du roman, on voit les soupçons converger sur Joseph K., qui finit par commettre l'imprudence habituelle des criminels, en retournant sur les lieux de son crime. L'arrestation au cours du troisième chapitre n'est alors qu'une conséquence logique de la faute et des maladresses de Joseph K. Au lieu d'une interrogation volontiers métaphysique sur la mystérieuse raison du Procès, le roman deviendrait celui de l'aveuglement d'un criminel, refusant d'admettre une culpabilité évidente au vu des éléments de l'enquête...

Le comportement ultérieur de Joseph K. s'accorde bien avec ce changement de l'ordre des chapitres. Il est loin d'être au-dessus de tout soupçon comme on le présente souvent. A de nombreuses reprises, il ment ou brave des interdictions pour parvenir à ses fins. Par exemple, pour trouver la salle d'audience du procès, il prétend qu'il recherche le domicile d'un menuisier nommé Lang. En faisant les yeux doux à la femme de l'huissier, il parvient à consulter les dossiers du juge et obtient le droit de pénétrer dans les archives judiciaires. Il abandonne son oncle à la sortie du domicile de l'avocat et il ne s'occupe pas de sa nièce, mais se laisse séduire facilement par des femmes légères et en fréquente régulièrement une, Elsa, serveuse de nuit dans un cabaret. Il dénonce les petits profits des inspecteurs venus l'arrêter, ce qui entraîne leur châtiment féroce. Autrement dit, il n'hésite pas à prendre des libertés avec les lois civiles et morales et à régler leur compte à ceux qui se mettent en travers de son chemin.

Le Procès n'est pas la seule œuvre de Kafka pour laquelle on puisse penser qu'une permutation de l'ordre des événements fait naître le sentiment d'étrangeté. On peut effectuer, par exemple, la même opération dans la Métamorphose. Au lieu de commencer par le réveil où Gregor Samsa s'aperçoit qu'il est transformé en scarabée, on peut mettre en tête de l'histoire le passage ultérieur où le gérant de l'entreprise se plaint en présence des parents de diverses irrégularités commises par Gregor: une allusion à des encaissements confiés depuis peu, ce qui laisse entendre qu'ils ont été détournés et un manque d'efficacité: «Sachez donc que votre travail de ces derniers temps ne nous a pas donné satisfaction; nous reconnaissons, je le veux bien, que la saison n'est pas propice aux grandes affaires, mais apprenez, Monsieur Samsa, qu'une saison sans affaire, cela ne peut, ne doit et ne saurait exister.» Dès lors, après cette permutation, la Métamorphose devient, comme le Procès un récit plus banal: renvoyé par son entreprise, Gregor s'enferme chez lui et fuit les contacts avec ses proches, car il ne peut plus assurer leur subsistance. Il se retranche du monde auquel il ne peut plus faire face, il se terre, il se protège par une carapace qui le rend insensible. La métaphore du scarabée s'impose alors assez naturellement et ne provoque pas la même horreur mêlée de stupeur que lorsqu'elle constitue l'événement initial. 


\section{DU PROCÉDÉ LITTÉRAIRE À LA CONCEPTION DU TEMPS HUMAIN}

Même en admettant que Kafka ait procédé de la manière que nous venons de supposer, créant par permutation des chapitres d'un récit assez banal, une atmosphère plus étrange et angoissante, on aurait seulement mis en évidence une technique littéraire, ce qui ne change pas la signification de l'œuvre. Or, le procédé de permutation va au-delà d'un simple artifice technique. Il est étroitement lié à la conception que Kafka se fait du temps, ou plutôt des différents temps humains. Les deux possibilités de roman avec et sans permutation continuent à coexister une fois l'œuvre fixée et achevée, c'est-à-dire que la séquence temporelle que doit suivre le roman reste en partie indéterminée. Sous le récit qu'il a finalement choisi, affleurent les autres récits possibles par permutation. On rejoint ici une expérience très profonde de Kafka, une expérience actualisée, illustrée et renforcée par l'extraordinaire développement de sa correspondance avec Felice Bauer, une expérience technique en quelque sorte.

Le 13 août 1912, Kafka rencontre à Prague, chez son ami Max Brod, une jeune Berlinoise, Felice Bauer, qu'il reconduit ensuite à son hôtel. Il commence avec elle une correspondance torrentielle qui durera cinq ans, jalonnés par deux fiançailles et leurs deux ruptures. Durant la première année, il adresse à Felice plus de trois cents messages. Lui réside à Prague, d'où il répugne à s'éloigner, et elle à Berlin qu'elle quitte rarement. Tous deux ont un emploi de cadre, et malgré leur âge vivent chez leurs parents. Comme il refuse d'utiliser le téléphone, ils s'écrivent. Le contrôle des échanges par correspondance se révèle être un véritable casse-tête. Un bon tiers des lettres de Kafka est consacré aux tentatives d'harmonisation des envois de courrier. Kafka et Felice utilisent quatre modes de courrier, les recommandés, les express, les lettres simples ou cartes postales et les télégrammes et ils ont quatre adresses possibles, leurs deux domiciles et leurs deux bureaux. Il s'en suit un cafouillage général, les télégrammes doublant les lettres, les arrivées au domicile devançant celles au bureau ou l'inverse selon l'horaire des levées et des distributions, les jours fériés différant en Allemagne et dans l'Empire austro-hongrois causent des déphasages supplémentaires. «Felice chérie, la poste se moque de nous, j'ai reçu ta lettre de mardi soir, et celle de la nuit de lundi que je me plaignais de ne pas avoir, voilà que je l'ai reçue ce matin au premier courrier. Il y a apparemment à l'intérieur de cet organisme postal si précis, un fonctionnaire diabolique qui joue avec nos lettres et les fait partir à son seul caprice, si encore il les faisait partir toutes!» ${ }^{4}$, ou encore: «Tu me laisses sans aucune nouvelle aujourd'hui mardi. Depuis dimanche $4 \mathrm{~h}$., je ne sais plus rien de toi, jusqu'à la distribution de demain, cela ne fera pas moins de 66 heures qui se remplissent tour à tour pour moi de tous les possibles bons ou mauvais $»^{5}$, ou ce moment de désespoir: «Lundi, tu m'as dit qu'à partir de maintenant, tu voulais m'écrire tous les jours. J'ai reçu cette lettre mardi, mercredi tu as eu ma réponse. Nous voilà vendredi soir et je n'ai pas encore un seul mot. Ne dois-je pas regretter que tu ne veuilles pas m'écrire 'par pitié', mais pour d'autres raisons, car si tu

\footnotetext{
Lettre du 28 novembre 1912.

5 Lettre du 24 décembre 1912.
} 
m'écrivais par pitié, j'aurais déjà la lettre depuis longtemps $»^{6}$. Parfois un facteur facétieux envoie une lettre de Felice à Vienne d'où elle met cinq jours à regagner Prague, ou un autre distribue un express au magasin des parents de Kafka. Parfois aussi, Kafka n'envoie pas immédiatement la lettre qu'il vient d'écrire ou en joint une écrite plusieurs mois auparavant. Parfois encore, il n'est pas dupe: «les lettres écrites ne se perdent pas, seules se perdent les lettres non écrites. $\gg^{7}$ On peut résumer ainsi cette expérience: soit deux personnes fixes, séparées par cinq cents kilomètres, correspondant par plusieurs moyens à différentes vitesses, et utilisant différentes adresses, comment faire se succéder dans le bon ordre les questions et les réponses?

Ni Kafka ni Felice ne trouveront la solution. C'est aussi pour Kafka la confirmation de l'incompatibilité des différents temps humains. Avant même que deux personnes se voient, avant même qu'elles se comprennent, il faudrait harmoniser leurs rythmes et c'est impossible. C'est aussi la raison de sa fascination pour les travaux de bureau dont il a plusieurs fois décrit la parfaite coordination. Le rapport de travail est inhumain et pourtant il est le seul à permettre la coordination de plusieurs personnes. En dehors de lui, les relations humaines ne sont pas en séquence. Il s'y mêle, à chaque échange, un souvenir d'échanges précédents et une anticipation des échanges à venir qui peuvent entièrement obscurcir le sens du message en cours et le situer logiquement avant ou après la date exacte de sa réception. Une bonne description de la communication sociale serait alors donnée par un tirage aléatoire du message à envoyer parmi plusieurs messages rédigés à une époque voisine. Le temps qui s'écoule entre deux messages n'est pas le même pour celui qui l'envoie et pour celui qui les attend. Inlassablement, Kafka tente d'introduire les règles formelles d'un temps universel, par exemple deux lettres par jour, une le matin postée à huit heures pour le domicile et l'autre à dix-huit heures adressée au bureau, ou une seule, ou une nécessairement le dimanche et liberté pour les autres jours. Il y ajoute le souhait d'un envoi d'une photo par mois. Il explique souvent à Felice que la régularité de leur correspondance importe plus que son contenu. Ils s'aiment, c'est un fait entendu, mais il est nécessaire qu'ils s'accordent en communiquant. Tous ces efforts de rationalisation échouent lamentablement.

Kafka a sans doute tiré les leçons de cette expérience de l'impossibilité d'une coordination entre deux individus, dans un petit texte intitulé Un incident quotidien $^{8}$ dont le début montre bien l'esprit: «A. doit régler une affaire importante avec B., de $\mathrm{H}$. Il se rend à $\mathrm{H}$. pour en discuter préalablement, y va et revient en dix minutes chaque fois et se vante chez lui de cette rapidité particulière. Le lendemain, il retourne à $H$., cette fois pour conclure définitivement l'affaire. Comme il peut prévoir que la décision exigera plusieurs heures, il part très tôt le matin. Mais bien que les circonstances accessoires soient absolument les mêmes - c'est du moins l'avis de A. -, cette fois, il met dix heures pour effectuer le même trajet. Quand, fatigué, il arrive à $\mathrm{H}$. le soir, on lui dit que B., furieux de son retard, est allé une demi-heure auparavant le trouver dans son village et que normalement, ils auraient dû se rencontrer en route. On conseille à A. d'attendre, mais A., inquiet

Lettre du 6 juin 1913.

Lettre du 7 juin 1913.

Un incident quotidien, Euvres complètes, op. cit., T. II, pp. 540-541. 
de son affaire, repart aussitôt et se hâte de rentrer. Cette fois, sans y prendre spécialement garde, il fait le trajet positivement en un clin d'œil. Chez lui, il apprend que B. est lui aussi venu très tôt - sitôt après le départ de A. - qu'il a même rencontré A. dans l'escalier, mais que A. a dit qu'il n'avait pas le temps maintenant et qu'il était pressé de partir.»

Hannah Arendt a fait un intéressant commentaire de ce passage qu'elle trouve exemplaire de la manière de Kafka9. Elle écrit: «D'un seul coup, tous les éléments essentiels qui ordinairement entrent en jeu en vertu d'un concours de circonstances malheureux se trouvent réunis : le zèle - A. part trop tôt, fait preuve de tant de hâte qu'il ne voit pas B. dans l'escalier; l'impatience - A. trouve le chemin étrangement long, ce qui a pour conséquence qu'il se soucie davantage du chemin que du but, à savoir rencontrer B. L'angoisse et la nervosité - suscitées par l'activité débordante et irréfléchie de A. qui revient, alors qu'il aurait attendre tranquillement le retour de B. .... C'est à partir de tous ces facteurs généraux et non à partir de l'expérience d'un événement particulier que Kafka construit l'incident.» Autrement dit, il n'y a aucune temporalité universelle dans cette histoire, mais un certain nombre de temporalités personnelles incompatibles entre elles qui sont juxtaposées dans un ordre dont l'importance reste secondaire, car ce qui compte, c'est la liste de ces «facteurs généraux » concentrés dans cette expérience particulière. La structure de l'événement n'est pas accessible par un compte-rendu objectif, minute par minute mesurées au temps universel, mais par la liste des durées personnelles de chaque action et décision de chaque individu. C'est aussi l'ensemble de ces éléments qui explique l'échec de la négociation entre A. et B. et non le déroulement objectif des opérations.

Il existe cependant un ordre formel des durées, et des actions des deux personnages, celui que Kafka a choisi en écrivant son histoire. Il part de deux évidences, la situation initiale dans laquelle A. et B. ne se connaissent pas et la situation finale où l'affaire échoue faute d'une coordination suffisante. Entre les deux, le récit file linéairement parce qu'il n'existe pas d'autre méthode de raconter et d'écrire que linéaire. Sa fonction n'est cependant pas de fournir une séquence d'événements se conditionnant les uns les autres, mais de passer par toutes les contraintes inhérentes au problème soulevé. La permutation des chapitres, la non-coïncidence des questions et des réponses dans la correspondance entre Felice et Kafka, le rendezvous manqué entre $\mathrm{A}$. et $\mathrm{B}$. pointent tous dans la même direction. Le temps doit être considéré comme un labyrinthe, c'est-à-dire comme une structure aux multiples détours, obligeant seulement à passer une fois et une seule par les diverses configurations retenues, ce qui est la définition même d'une permutation. Quelle permutation choisir, pourquoi telle séquence plutôt qu'une autre? Questions relativement secondaires. Il faut seulement que le récit passe par toutes les étapes et en fasse sentir la nécessité. En ce cas, le récit le plus étrange retiendra mieux l'attention que le plus banal qui peut insidieusement faire sauter des étapes, c'est-àdire des éléments de la permutation. De ce point de vue, la ligne droite est plus trompeuse que le labyrinthe. Elle propose la solution la plus simple et la plus banale, sautant les contraintes les plus cachées. Au contraire, la perturbation de la

9 Franz Kafka: a Re-evaluation, in «Partisan Review», 11/4, Octobre 1944, pp. 412-422, traduit dans: Hannah Arendt: la tradition cachée, C. Bourgois, Paris, 1987. 
droite permet de faire le détour par les «facteurs généraux ». Le labyrinthe du temps signifie l'exhaustivité, tandis que le parcours direct signifie l'oubli des contraintes à l'exception des conditions initiales et finales. Par ses permutations Kafka plonge donc le lecteur dans le labyrinthe du temps. Dans celui de l'espace aussi pour une autre raison.

\section{LABYRINTHE DU RÉCIT}

L'analyse d'H. Arendt ne vaut pas seulement pour le court récit de la rencontre manquée entre A. et B. Elle ne justifie pas seulement le changement possible d'ordre des premiers chapitres ou passages du Procès et de la Métamorphose, mais elle concerne les grandes œuvres de Kafka. Chacune d'entre elles constitue une réflexion serrée sur l'ensemble des possibilités d'une question donnée. Contentons-nous de le montrer ici pour les deux premiers romans, l'Amérique et le Procès.

L'Amérique est consacré à un problème simple: comment un jeune homme peut-il se dégager du cercle familial. Kafka passe en revue quatre configurations qui correspondent à toutes les combinaisons possibles de deux critères: formation/vie active d'une part, et travail à l'intérieur de la famille/ famille à l'intérieur du travail d'autre part. Dans le cas de la formation, les deux possibilités sont constituées par la famille de Karl Rossmann qui le chasse et par l'éducation que l'oncle lui fait donner dans son entreprise de New York. Les deux échouent de la même manière sur une agression brutale de Karl par des femmes, la domestique de ses parents qui le viole, raconte-t-il, et Clara, la fille de l'ami de son oncle, qui l'immobilise par une prise de judo et menace de le rosser sévèrement.

Dans les deux possibilités offertes par la vie active, Karl échoue aussi lamentablement car il se réinsère chaque fois dans une parodie de famille. Engagé comme liftier à l'hôtel Occidental, il devient une sorte de fils protégé par la cuisinière en chef dont l'amant est le chef du personnel. Une autre protégée de la cuisinière, Thérèse, avec laquelle il pouvait avoir une liaison est réduite à un rôle de sœur. La seconde situation se déroule dans l'appartement de la cantatrice Brunelda où il a abouti après son licenciement de l'hôtel. Tandis qu'on le force à devenir domestique, une famille fictive se reconstruit autour de lui avec Brunelda dans le rôle maternel, son amant Delamarche dans celui du père tyrannique et le faible ami de Delamarche, Robinson, faisant figure de frère. Karl, quelle que soit la situation ne peut donc échapper à sa détermination de fils et à la reconstitution de bric et de broc d'une configuration familiale. La relation de filiation s'est transformée en état immuable de fils.

Dans le Procès, Kafka aborde une autre question familiale qui l'obsède à titre privé: comment se marier ou se mettre en couple. La structure du roman est plus élaborée que celle de l'Amérique, car, au second degré, Kafka se demande si certains savoirs peuvent répondre à sa question. Il mène donc son enquête à deux niveaux, celui de l'expérience personnelle et celui de la connaissance. En matière d'expériences féminines, le héros Joseph K. passe par tout le spectre des compagnes possibles: demi-mondaine avec Elsa, qu'il rejoint une fois par semaine après sa nuit de cabaret, femme moderne et libérée avec $M^{\text {lle }}$ Bürstner, femme d'intérieur, avec l'épouse de l'huissier qu'il rencontre en train de faire la lessive, 
ou de transformer les locaux de la justice en intérieur bourgeois, femme-maîtresse avec l'infirmière Léni qui le séduit. Il y ajoute les possibilités de pédophilie (les petites filles perverses de l'atelier du peintre Titorelli), d'homosexualité (le chapitre inachevé sur Titorelli) et de célibat ecclésiastique (rencontre à la cathédrale avec l'aumônier des prisons).

Ce passage en revue des possibilités pratiques est coiffé par la recherche d'un savoir théorique sur la question. La plus grande part du roman est en effet consacrée à des discussions serrées de Joseph K. avec trois personnages représentant les trois types de savoir mobilisables, l'avocat Huld, pour la sagesse populaire, le peintre Titorelli, pour le savoir scientifique ou la connaissance positive et l'aumônier pour la religion. On sait qu'aucun des trois ne sera capable d'apporter une réponse ni de s'écarter de l'ordre établi. Ni les expériences pratiques, ni les connaissances théoriques n'ont donc pu éclairer Joseph K. sur sa question.

\section{LE FIL DU RÉCIT}

La difficulté de tels romans dont les scènes ou stations sont bien définies consiste à les articuler de manière exhaustive avec un minimum de suspense. Il faut passer par toutes les possibilités requises, tout en donnant l'impression d'un récit. Il faut donc introduire un ressort romanesque pour justifier l'ordre d'exposition adopté alors qu'a priori, tous les ordres sont également valables puisque il s'agit seulement de balayer un ensemble de possibles. Systématiquement, Kafka a utilisé comme ficelle dramatique le dépérissement de son héros, de moins en moins agressif, de moins en moins assuré, de moins en moins bien installé dans la vie, de plus en plus fatigué à mesure que le roman progresse. Kafka peut ainsi choisir un chemin qui passe par toutes les possibilités qu'il désire examiner. Ses romans constituent donc bien des labyrinthes qui passent par toutes les scènes prévues tout en ménageant une progression minimum du récit avec l'affaiblissement régulier du héros après chaque épreuve, comme un boxeur encaissant de plus en plus durement les coups avant le KO final.

Cette méthode reprend un procédé narratif traditionnel et même très ancien. On peut par exemple la rapprocher de celle de l'Odyssée, où, selon l'argument de P. Vidal-Naquet, Homère aurait cherché simplement à prospecter tous les cas possibles de non-humanité caractérisés par l'anthropophagie et l'absence de culture du blé et de la vigne ${ }^{10}$. De même, l'Iliade peut être lue comme un manuel de combat exposant les diverses positions d'attaque et de riposte. Plus près de notre époque, E. Mâle ou E. Panofsky ont montré comment les grands monuments gothiques pouvaient s'interpréter comme un exposé savant des règles de la scolastique, comme un code et comme un mode de déduction. Ces références s'accordent aussi avec les techniques anciennes de mémorisation dites des «arts de mémoire ${ }^{11}$. Par exemple, un avocat qui devait mémoriser une plaidoirie en mettait en scène les différents éléments en les situant successivement dans les pièces d'une villa qu'il visitait par l'imagination.

\footnotetext{
10 P. Vidal-Naquet, Le chasseur noir, Paris, La Découverte, 1985.

11 France Yates, The Art of Memory, trad. fr. L'art de la mémoire, Paris, Gallimard, 1974.
} 
Il y a cependant une différence importante entre ces derniers exemples et la courte histoire de A. et B. ou l'échange de courrier entre Kafka et Felice: le labyrinthe ou la permutation ne résulte plus d'un désaccord des temporalités personnelles, mais du mouvement d'un temps reconstruit, celui du vieillissement ou de l'épuisement progressif du héros, qu'il s'agisse d'Ulysse, de Karl, de l'arpenteur ou de Joseph K., ou celui de la visite dans le cas des arts de la mémoire. Le labyrinthe inchoatique de la juxtaposition des temporalités est reconquis personnellement par le héros non pas civilisateur, mais ordonnateur. L'arbitraire de la succession des scènes est organisé sur le modèle d'une quête. Ni Ulysse, ni les chevaliers de la table ronde ne changent au cours de leur quête, ils vieillissent seulement. De même, on a souvent remarqué que les héros de Kafka ne changeaient absolument pas dans leur façon d'agir d'un bout à l'autre des romans, mais vieillissaient à vitesse accélérée. Karl reste aussi naïf malgré les épreuves, Joseph K. aussi raisonneur, malgré l'incohérence de la justice et l'arpenteur continue de réclamer son dû en dépit de tout ce qu'il apprend sur l'inutilité de ses démarches. On pourrait ajouter que Gregor continue imperturbablement à faire le scarabée.

\section{LA NATURE DU LABYRINTHE}

Dans tous les cas précédents, le labyrinthe ne signifie donc pas un égarement dans un lieu dont on ne trouve pas la sortie, mais un cheminement dans un sentier tortueux. C'est toute la différence entre le labyrinthe et le dédale. Dans un labyrinthe, dès qu'on commence à avancer, on est sûr d'atteindre la sortie. Il n'y a qu'un seul chemin possible, mais il accumule les détours qui vous donnent l'impression d'être perdu. Dans un dédale au contraire, il faut choisir à chaque croisement son chemin si bien qu'un grand nombre d'itinéraires sont possibles dont la plupart ramènent sur vos pas ou aboutissent à un cul-de-sac. Le dédale n'a pas grande portée métaphorique car il signifie seulement une certaine confusion de l'état du monde, tandis que le labyrinthe possède une forte tension du fait de son apparente contradiction. A la fois on est perdu et on est sûr d'arriver à la sortie, à la fois l'entrée et la sortie sont proches et le cheminement de l'une à l'autre éloigné. A la fois on est à l'intérieur et à l'extérieur, car parler de labyrinthe n'est possible qu'en confrontant la complexité de son tracé avec la simplicité du plan euclidien dans lequel il s'inscrit. Le labyrinthe est un intermédiaire entre la droite et le plan, entre les dimensions un et deux. En termes modernes, il s'apparente à un fractal, justement de dimension intermédiaire. De très beaux exemples de fractals, comme les courbes de Sierpinski remplissant tout le plan, donnent d'ailleurs l'impression de labyrinthe. Le labyrinthe s'oppose autant à l'ordre linéaire strict de la droite qu'à l'absence d'ordre des points d'un plan. Il fabrique un ordre différent de celui des distances habituelles, éloignant des points très proches, mais en laissant d'autres à leur distance habituelle selon les détours de son cheminement.

De tels labyrinthes ont été construits par la plupart des civilisations. Ils figurent sur des pièces de monnaie crétoises, sur des pierres gravées de runes, sur les dessins des Indiens d'Amérique. On les retrouve sur le dallage de l'entrée des cathédrales gothiques et dans les sculptures du Ramayana. En dehors de leurs aspects initiatiques sur lesquels on insiste fréquemment, ils ont une fonction formelle importante. Si l'on définit en effet les formes comme des interfaces ou des 
solutions de continuité ou des tracés régulateurs entre l'esprit ou les idées, et le monde matériel, les labyrinthes sont l'outil de jonction des formes différentes. Simultanément ils en respectent la différence en donnant l'impression d'une grande distance, et ils en assurent la continuité par connexion. Dans le domaine physique, on rencontre fréquemment des labyrinthes à l'interface de formes différentes. Par exemple, les transitions de phase entre deux états de la matière, solide et liquide ou liquide et gazeux par percolation impliquent une extension importante de la surface de contact des deux phases, au moyen de structures labyrinthiques. L'intestin est un autre exemple d'une telle surface de transition. De nombreux processus catalytiques prennent aussi la forme de labyrinthes pour la même raison. Logiquement d'ailleurs, à partir du moment où l'on distingue des formes simples et tranchées, on ne peut les relier que par d'autres formes dont le principe est différent, des formes donc complexes et moins tranchées.

En assurant la transition entre entités distinctes, le labyrinthe crée une impression de nécessité dans le passage d'une forme à une autre. Ainsi, non seulement le chemin qui passe par les différentes situations que le roman ou l'épopée doit parcourir forme-t-il un labyrinthe dans son ensemble, mais aussi, la connexion entre deux situations distinctes, comparables à deux formes différentes, est-elle assurée par un labyrinthe qui assume donc l'absence de dépendance causale entre scènes successives. Ce peut être l'errance sur une mer déchaînée entre deux étapes de l'Odyssée, ou entre deux îles visitées par le capitaine Gulliver. Dans les romans de Kafka, la méthode est plus raffinée. Kafka crée de véritables labyrinthes matériels entre les différentes situations qu'il juxtapose. Le héros passe d'une scène à la suivante par une série de détours dans lesquels il se croit perdu avant de déboucher souvent à l'endroit exact qu'il cherchait à atteindre ou qu'il devait atteindre. Dans l'Amérique, Karl, après s'être fourvoyé dans le labyrinthe des coursives et avoir rencontré un inquiétant soutier, se retrouve subitement dans la cabine du capitaine où son oncle l'attend. De même, après avoir erré dans la cathédrale enténébrée, Joseph K. tombe nez à nez avec l'aumônier qui l'attendait sans qu'il le sût.

Ces transitions par des labyrinthes font référence à échelle réduite au labyrinthe d'ensemble que constitue le roman entier. Les labyrinthes réels entre deux lieux de deux scènes successives constituent ainsi la métonymie de l'ensemble de la démarche littéraire de Kafka. Ils rappellent que malgré leur absence de direction, ils conduiront bien à l'objectif, c'est-à-dire, que le roman passera bien par toutes les configurations imaginables de la question posée, qu'il s'agisse de la sortie de la famille dans le cas de l'Amérique ou de la rupture du célibat dans celui du Procès.

Pour accentuer la ressemblance entre les labyrinthes locaux qui raccordent les scènes différentes du roman et le labyrinthe d'ensemble du roman, Kafka y épuise son héros de la même manière, mais en accéléré. Par exemple, dans la maison Pollunder, Karl se perd dans d'étranges couloirs obscurs, puis ne retrouve son chemin que pour recevoir le mot de son oncle qui le chasse et s'enfuir à bout de souffle par le jardin dans la nuit, menacé par le jappement des chiens de garde qu'il imagine à ses trousses. A l'issue de leurs recherches, les héros doivent souvent gravir d'impressionnants escaliers, que ce soit pour gagner l'appartement de Brunelda ou pour atteindre l'atelier de Titorelli. Pour corser la difficulté, Kafka joue aussi sur le temps et sur les possibilités de mobilité. Cela est particulièrement net dans le Château, où l'arpenteur, lors de sa première sortie de l'auberge, ne parvient pas à 
se rapprocher du château qu'il aperçoit, s'enfonce de plus en plus dans l'épaisseur de la neige et voit le jour tomber alors qu'il a l'impression de s'être mis en route tôt le matin, il y a peu de temps. Bien qu'il n'y ait pas de changement de lieu dans la Métamorphose, c'est aussi des difficultés de locomotion du scarabée que vient le ralentissement et la sorte d'engourdissement des mouvements. Gregor ne peut plus passer facilement par la porte de sa chambre. Il doit effectuer un mouvement de rotation difficile pour tourner et ne parvient pas à contrôler sa marche arrière.

Le labyrinthe ne distord pas seulement l'espace mais le temps. De même qu'il éloigne des lieux proches en y introduisant la distance de ses circonvolutions, il sépare des instants voisins en y intercalant des durées anormales. On remarque que tous ces éléments sont présents dans la petite histoire Un étrange incident que nous avons commentée plus haut: accélérations et freinages du temps, escaliers, pénombre, déplacements.

Paradoxalement, les transitions par les labyrinthes se révèlent plus favorables aux héros que les scènes statiques. Dans les labyrinthes, les héros semblent avoir ramassé et ressaisi leurs forces et ils en émergent en meilleure condition qu'ils ne s'y sont engagés. En revanche, les scènes qui leur succèdent apparaissent comme des points de désordre et d'agressivité concentrés dans le temps et l'espace. Elles se déroulent le plus souvent dans un espace dont l'exiguiité renforce l'oppression et sur une durée courte que l'on voit fuir à toute vitesse. Ainsi de la scène d'amour de l'arpenteur avec Frieda sous le comptoir, de sa chambre à l'auberge du pont, de la pièce où K. rencontre un conseiller du château. Ainsi aussi de la chaire trop étroite de l'aumônier, des locaux exigus et oppressants de la justice où Joseph K. étouffe, du réduit où sont châtiés les inspecteurs qui lui ont signifié son arrestation. Sans avoir connu Lewis Caroll, Kafka recourt souvent aux mêmes procédés que l'auteur d'Alice au pays des merveilles.

\section{L'ÉTUI PROTECTEUR DU LABYRINTHE}

Le labyrinthe n'est donc pas exactement une métaphore de l'existence. Il représente plutôt les contraintes que ses parois imposent à cette existence qu'elles protègent du temps et de l'espace extérieurs en la canalisant. Le cheminement, image plus habituelle de l'existence, est au contraire représenté par le fil d'Ariane, ou par les cailloux du petit Poucet, c'est-à-dire par l'intérieur du labyrinthe, par le chemin praticable entre les parois. Le labyrinthe kafkaïen constitue une condition nécessaire de l'existence. Il maintient l'unité de la personnalité toujours menacée d'éclatement en plusieurs personnages sous les traits desquels l'auteur se peint souvent dans de petits récits. La division du moi et son risque afférent de dissolution ne sont pas une maladie propre à Kafka mais menacent tout humain. Dans une lettre à Gretl Bloch, Kafka écrit de Felice, dont il vante pourtant la solidité: «Je connais F. sous la forme de quatre jeunes filles presque inconciliables entre elles, qui me sont presque également chères. La première... La deuxième est celle qui m'a écrit des lettres (celle-là était multiple en elle-même, mais néanmoins douée d'unité). La troisième.... ${ }^{12}$. On sait d'ailleurs par sa correspondance que Kafka s'imposait de

12 Lettre à Gretl Bloch, 10 novembre 1913. 
nombreuses contraintes d'emploi du temps, d'alimentation - il était végétarien -, ou de gymnastique - il était adepte de la méthode müllerienne qu'il recommanda à Felice -, sans oublier ses tentatives d'organiser l'échange de lettres avec sa bienaimée $^{13}$. Peut-être aussi est-ce l'origine du labyrinthe crétois. Créé pour enfermer le Minotaure ${ }^{14}$, il aurait en fait eu pour but d'empêcher cet être double, homme et taureau, de se dissocier et donc de se détruire à l'extérieur. Le labyrinthe protégeait le Minotaure plus qu'il ne le gardait prisonnier. Réduit à l'extrême, le labyrinthe devient la coquille chitineuse du scarabée qui a remplacé le corps de Gregor ${ }^{15}$.

Tant que l'on est à l'intérieur du labyrinthe, on reste en vie. Si l'on s'échappe, comme repris par le principe d'inertie galiléen, on court en droite ligne à sa perte rapide. Ainsi, dans la courte et parfaite histoire du Verdict, maudit par son père, Georges Bendemann dévale les marches de l'escalier «comme sur un plan incliné », «jaillit hors de la porte et franchit les rails du tram, poussé irrésistiblement vers l'eau », saute le parapet du pont et se jette dans le fleuve (plus exactement, il «chute» comme s'il était mû de l'extérieur et non soumis à sa propre volonté). De même, dans l'Amérique, Karl, qui cherche à échapper aux policiers qui le poursuivent, en dévalant en ligne droite un grand boulevard, au moment où il va être rattrapé, est secouru par Delamarche qui l'a suivi en parallèle par les passages et les cours intérieures et qui le sauve in extremis en l'entraînant dans le même labyrinthe. Le récit Souvenir du chemin de fer de Kalda met en scène cette irrévocable malédiction de la ligne droite puisque ledit chemin de fer, qui traverse la Sibérie, n'atteint même pas la ville de Kalda. Le héros, responsable d'une gare, y dépérit rapidement, comme si les rails rectilignes et parallèles l'avaient directement mené à la fin de son existence. Il ne peut survivre faute d'être protégé par un labyrinthe.

Un autre texte court, datant de la jeunesse de Kafka, propose la même idée sous une forme un peu différente. Dans Hé, dis-je, un jeune homme discute avec un célibataire endurci. Ils comparent l'existence à un cercle dans lequel se rejoignent passé et futur: «Ce cercle nous appartient, mais seulement tant que nous le tenons; que nous nous écartions de lui une seule fois, par suite de quelque absence, d'une distraction, d'une frayeur, d'un étonnement, d'une lassitude, et déjà il est perdu dans l'espace, nous avions jusque-là le nez plongé dans le fleuve du temps, nous reculons maintenant, nageurs passés, promeneurs actuels, et nous sommes perdus $»^{16}$. Le labyrinthe est certes une situation terrible à vivre, mais il

13 L'inquiétude quant à l'existence et surtout quant à la cohérence de la personnalité traverse d'ailleurs presque toute la littérature allemande du début de siècle, de Thomas Mann à l'Homme sans qualités de Robert Musil.

14 Référence au récit de Dürrenmatt, qui a servi de référence pendant les travaux du colloque et est résumé dans la contribution de Marie-Jeanne Borel parue en introduction au présent numéro de la Revue: Friedrich Dürrenmatt, La mort de la Pythie, suivi de Minotaure, Lausanne, L'Age d'Homme, 1990.

15 Dans la courte histoire Préparatifs de noces à la campagne, écrite peu avant la Métamorphose, ces thèmes s'entrecroisent. L'auteur, qui se rend chez sa fiancée, progresse très difficilement, freiné par le mauvais temps et la mauvaise volonté des transporteurs. Il s'imagine dissocié, son esprit voyageant et son corps restant au lit avec « la silhouette d'un gros coléoptère, d'un lucane, d'un hanneton, je crois.... je presse mes petites pattes contre mon abdomen» (CEuvres complètes, T. II, pp. 83-84). 
représente notre existence et notre chance de la posséder personnellement, à l'écart des rencontres et des tentatives de communication sans succès avec autrui. Si l'on quitte son cercle, on plonge et l'on se noie. Dans un autre récit assez long et tardif, le Terrier $^{17}$, Kafka décrira d'ailleurs le labyrinthe des galeries dans lesquelles vit une sorte de taupe et son angoisse d'être dérangée par le plus humble vermisseau fouisseur ${ }^{18}$.

La métaphore du labyrinthe possède ainsi dans les récits de Kafka deux statuts différents. Elle est la forme et la garantie du récit - exhaustivité des combinaisons étudiées - et elle est la forme et la garantie de l'existence des personnages principaux. La connexion entre les deux statuts s'effectue par le fil du récit qui devient celui de l'existence du héros, et par les transitions entre scènes qui se matérialisent en labyrinthes réels. Par son premier statut, la métaphore du labyrinthe est recherche de l'objectivité scientifique et par son second, ultime refuge de la subjectivité. La tension qui en résulte rappelle celle des contes, dont Kafka était lecteur, mais en l'amplifiant. On peut le montrer à titre d'exemple, en rapprochant un conte d'Andersen, le Briquet ${ }^{19}$, de la parabole de la sentinelle que l'aumônier raconte à Joseph K. dans le Procès.

Dans le conte d'Andersen, une sorcière indique à un soldat un labyrinthe qui permet d'entrer dans une caverne dont les trois chambres successives sont gardées par des chiens de plus en plus gros, le dernier ayant « des yeux grands comme des tours ». En actionnant le briquet que la sorcière lui a donné, le soldat neutralise les trois chiens et s'empare du trésor qu'ils gardaient. Il ressort du labyrinthe, tue la sorcière, et après de nombreuses aventures, épouse la fille du roi. Dans la parabole du Procès, une sentinelle comme la sorcière garde l'entrée qui donne l'accès à la «loi», mais elle dissuade le paysan d'entrer en lui disant que dans chaque chambre qu'il traversera, il trouvera une sentinelle encore plus terrible, la troisième ne pouvant même pas être regardée. En parlant, la sentinelle joue avec une blague à tabac. Le paysan n'ose pas entrer, vieillit en cherchant à fléchir la sentinelle sans succès et peu avant de mourir, aperçoit une forte lueur à l'intérieur. Les éléments communs au conte d'Andersen et à la parabole sont trop nombreux pour qu'il s'agisse d'un hasard: briquet et tabatière, troisième chambre avec le chien terrible (chien de garde) et avec la sentinelle terrible (de garde aussi), lueur entrevue par le paysan et lueur du briquet. Mais le traitement par Kafka diffère entièrement de celui d'Andersen. Le paysan intériorise le labyrinthe car en ne s'y engageant pas personnellement, il se condamne. Passé à côté du «cercle», il est perdu pour reprendre l'image du récit Hé, dis-je. Au contraire, le soldat d'Andersen pille et tue conformément à sa nature de soldat.

17 CEuvres complètes, T. II, pp. 719-772.

18 Cette conception chtonienne du labyrinthe s'oppose terme à terme à la description que F. Dürrenmatt a donnée du mythe du Minotaure dans la nouvelle (citée note 14). Les parois du labyrinthe crétois sont translucides et, comme il est en plein air, inondées de soleil et de reflets incandescents. Dans cet embrasement général, les personnages se multiplient, précipitent leur course, leur peur, leur soif de tuer et le sang dégouline. La conception solaire du labyrinthe rappelle les enceintes de confinement atomique et les cyclotrons à l'intérieur desquels les particules accélérées atteignent des vitesses et des températures inouïes. Au contraire, les labyrinthes sombres de Kafka préservent la vie de ses héros. Leur enveloppe les protègent contre les dangers du monde sur lequel ils mènent leurs observations et leurs expériences.

19 Andersen, Contes, Paris, La Pléiade. 
La parabole de la sentinelle résume à elle seule les usages successifs de la métaphore du labyrinthe: elle constitue l'une des configurations par lesquelles le récit devait passer, celle qui montre que la religion en tant que savoir recommande la soumission dans des termes analogues à ceux de la tradition et de la science. La métaphore réalise ce détour grâce à un décalage des temporalités, le paysan consumant une vie entière à attendre, au sein d'une conversation de quelques minutes entre l'aumônier et Joseph K. Dans la parabole, un labyrinthe réel apparait ensuite, situé derrière la porte de la Loi, et enfin ce labyrinthe est intériorisé par le paysan en destin qui confine et bloque sa vie entière. Le conte d'Andersen n'utilise qu'une opération. Il objective sous forme de passage par un labyrinthe, un enrichissement mystérieux du soldat. Le récit de Kafka pousse le mécanisme bien plus loin avec un effet en retour du labyrinthe sur le paysan. La technique de Kafka est ainsi d'une grande subtilité. Il représente la possibilité d'une autre science sociale, aussi rigoureuse sinon plus que celle qui domine actuellement, mais s'exprimant grâce à la littérature et non par le truchement de modèles des sciences physiques ou biologiques. Le Procès, l'Amérique, la Métamorphose et ces courts récits que nous avons croisés, mettent en scène chacun un véritable problème social et le traitent en détail. Oui, seules certaines formes familiales sont stables et on n'y échappe pas, oui l'organisation du travail ne peut pas se transposer à celle de la société, oui le désordre des foules manifeste l'absence d'un principe d'organisation supérieur. Au lieu de l'exprimer par des tableaux statistiques, par l'exploitation d'entretiens directifs ou non, par des lois du comportement, par des analogies avec les organismes, les tissus, ou la croissance biologiques, Kafka utilise une méthode que plus tard et dans un autre cadre, $\mathrm{H}$. Arendt ${ }^{20}$ a décrite en trois étapes: précompréhension, analyse, restitution. Pour cela, Kafka passe par le filtre de la littérature et dès lors trouve dans la métaphore un outil essentiel, alors que dans les sciences sociales qui ont adopté le langage de la physique et de la biologie, la métaphore est considérée comme un résidu préscientifique et une scorie $^{21}$.

\section{Ecole des hautes études des sciences sociales}

Paris

20 H. Arendt, La nature du totalitarisme, Paris, Payot, 1985.

21 Le fait que Kafka ait eu pour directeur de sa thèse de doctorat Alfred Weber, le frère de Max, a pu avoir une influence sur sa manière «sociologique». Il ne faut pas non plus oublier la configuration littéraire de l'Allemagne au début du siècle. Le roman ne s'y opposait pas aux sciences de la nature, mais à la poésie, dans laquelle Stefan Georg et son puissant cercle mettaient tous les espoirs de progrès. Pour eux, seule la poésie pouvait comprendre le présent et donner une idée de l'avenir, tandis que les essais et le roman ne parlaient que d'un passé déjà mort. Thomas Mann, Musil, Kafka prônaient une littérature du sens contre une poésie de l'instinct. Alors qu'en opposant ici Kafka aux physiciens sociaux, nous paraissons en faire un prosélyte d'une approche intuitive et peu rationnelle, il occupait la place contraire à son époque et dans son milieu. L'intéressant ouvrage de W. Lepenies est une bonne introduction à ces débats allemands (Les trois cultures, Paris, Ed. de la MSH, 1990) ainsi que l'étude de Fritz Stern (Politique et désespoir, Paris, Le Seuil, 1992). 